\title{
Tecnologia assistiva para a promoçáo de atividades da vida diária com crianças em contexto hospitalar
}

\author{
Adriana Moniz da Silveira ${ }^{a}$, Regina Helena Vitale Torkomian Joaquim ${ }^{\text {, }}$ \\ Daniel Marinho Cezar da Cruz ${ }^{c}$
}

\author{
aTerapeuta Ocupacional, Universidade Federal de São Carlos - UFSCar, São Carlos, SP, Brasil

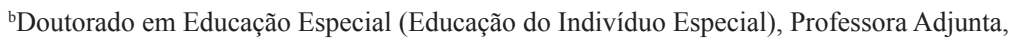 \\ Universidade Federal de São Carlos - UFSCar, São Carlos, SP, Brasil \\ 'Doutorado em Educação Especial (Educação do Indivíduo Especial), Professor Assistente, \\ Universidade Federal de São Carlos - UFSCar, São Carlos, SP, Brasil
}

\begin{abstract}
Resumo: Teve-se por objetivo caracterizar a rotina de uma enfermaria pediátrica no que se refere ao desempenho de seus usuários, nas AVD: higiene pessoal, banho e alimentação; e apontar os possíveis usos da tecnologia assistiva referentes ao desempenho dessas AVD. Participaram da pesquisa 10 membros de uma equipe de enfermagem, responsáveis pelos cuidados das crianças; 12 crianças internadas na instituição hospitalar de seis a doze anos; e seus respectivos pais ou responsáveis. A caracterização da enfermaria pediátrica deu-se por meio de entrevistas semi-estruturadas e roteiros de observação, elaborados pelos pesquisadores. Como resultados, tem-se que $100 \%$ $(n=10)$ dos profissionais, membros da equipe de enfermagem referiram realizar orientação e/ou supervisão e/ou execução das AVD de higiene pessoal, banho e alimentação das crianças sob sua responsabilidade. Entre as crianças participantes, o tempo de internação variou de três a quarenta e cinco dias; $75 \%(n=9)$ referiram dependência na AVD de higiene pessoal, 83,3\% $(\mathrm{n}=10)$ na atividade de banho e $16,6 \%(\mathrm{n}=2)$ na atividade de alimentação; as crianças relatam também que seus acompanhantes são quem as ajuda nas AVD de higiene pessoal e/ou banho e/ou alimentação. Os responsáveis pelas crianças participantes reportam que houve diferença no modo de execução da atividade de higiene pessoal $(41,6 \%, \mathrm{n}=5)$, de banho $(66,6 \%, \mathrm{n}=8)$ e de alimentação $(50 \%, \mathrm{n}=6)$ das mesmas. Embora com pequenas diferenças dos relatos das crianças, os pais reportaram dados similares, sendo o banho a atividade com maior porcentagem de ajuda. A partir desses resultados são apontadas recomendações acerca do uso da tecnologia em contexto hospitalar.
\end{abstract}

Palavras-chave: Terapia Ocupacional, Equipamentos de Autoajuda, Atividades Cotidianas, Autonomia Pessoal.

\section{Assistive technology for the promotion of activity of daily living with children in hospital contexts}

\begin{abstract}
The purpose of this research was to characterize the routine of a pediatric nursing ward in relation to the performance of their users on the Activity of Daily Living - ADL: personal hygiene, bathing and feeding, as well as point out the possible uses of assistive technology concerning the ADL performance. The participants were the following; 10 members of a nursing team, 12 caretakers and their hospitalized children with ages between six and twelve. The characterization of the pediatric nursing was made through semi-structured interviews and observation developed by the researchers. Results showed that $100 \%(n=10)$ of professionals, members of the nursing staff, reported performing guidance and/or supervision and/or performance of the following ADL: personal hygiene, bathing and feeding of children under their responsibility. Children remained in hospital from three to forty-five days and $75 \%(n=9)$ of them reported ADL dependence in personal care, $83.3 \%(n=10)$
\end{abstract}

Autor para correspondência: Regina Helena Vitale Torkomian Joaquim, Centro de Ciências Biológicas e da Saúde, Universidade Federal de São Carlos, Rod. Washington Luis, Km 235, CEP 13565-905, São Carlos, SP, Brasil, e-mail: regin@ufscar.br 
in the bathing activity and $16.6 \%(n=2)$ in the feeding activity. Children also reported that they were helped with personal care, bathing and feeding by their caretakers. Caretakers identified differences in the performance of the children's personal hygiene $(41.6 \%, \mathrm{n}=5)$, bathing $(66.6 \%, \mathrm{n}=8)$ and feeding $(50 \%, \mathrm{n}=6)$ activities. Except for slight differences in the reports of the children, parents reported similar data and the bathing activity presented the highest level of assistance. Recommendations on the use of technology in hospital contexts were elaborated based on these results.

Keywords: Occupational Therapy, Self-help Devices, Activity of Daily Living, Personal Autonomy.

\section{Introdução}

A infância caracteriza-se por um período importante para o desenvolvimento de aspectos motores, cognitivos e psicossociais do ser humano (BORTOLOTE; BRÊTAS, 2008), sendo o crescimento e o desenvolvimento eixos referenciais para as atividades de atençáo à criança e ao adolescente (BRASIL, 2002). Deste modo, tem-se que o desenvolvimento permite à criança adquirir habilidades que auxiliam no seu processo de independência (BEE, 1997). Referindo-se ao desenvolvimento saudável Takatori, Oshiro e Otashima (2004) afirmam a ocorrência de encontro e interação dos processos maturacionais herdados pela criança, com os efeitos facilitadores e preservadores do ambiente externo.

Ao conjunto de condiçóes biológicas, existem diversas variáveis ambientais que podem atuar como fatores agravantes ou atenuantes para a promoção do desenvolvimento (LINHARES et al., 2004). Segundo o modelo socioecológico, postulado por Bronfenbrenner (1996), ocorrem processos de interação entre a criança e seus contextos desenvolvimentais, representados por estruturas interdependentes que "atuam" na promoção de seu desenvolvimento.

Grigolatto et al. (2008) observam que quando existem alteraçôes comprometedoras das funçóes inerentes ao indivíduo, tais como infecçóes, traumas, malformaçōes, dentre outras, há a interferência na fluidez da cadeia desenvolvimentista. Motta e Takatori (2001) adicionam que quando ocorrem alteraçóes no desenvolvimento, a possibilidade de atingir a maturaçáo de uma vida saudável depende, dentre outros fatores, da realização de atividades que compóem o dia a dia do sujeito.

As Atividades da Vida Diária (AVD), também conhecidas como atividades pessoais da vida diária ou atividades básicas da vida diária, correspondem às atividades relacionadas aos cuidados pessoais $\mathrm{e}$ mobilidade (TEIXEIRA, 2003). As AVD incluem atividades de higiene pessoal e autocuidado, banho, alimentação, vestuário, controle de esfíncteres, mobilidade funcional, cuidado com equipamentos pessoais, atividade sexual, uso de vaso sanitário, dormir e descansar, além da atividade de comunicação (MELLO; MANCINI, 2007; TEIXEIRA, 2003).

Segundo Takatori, Oshiro e Otashima (2004) o lar é um ambiente adequado para um desenvolvimento saudável na infância, pois é o local onde ocorrem as primeiras experiências da criança envolvendo segurança e confiança, permitindo assim a exploração e a experimentação de diferentes relacionamentos. $\mathrm{Na}$ ocasião de uma doença que leve à hospitalização, a criança passa a viver em um ambiente desconhecido ou acrescenta o novo espaço ao seu cotidiano (TAKATORI; OSHIRO; OTASHIMA, 2004). Ocorre uma quebra de sua rotina, de modo que essa criança necessita se adaptar à nova realidade, que por sua vez inclui exames, procedimentos dolorosos, horários, visitas restritas, etc (PEDROSA et al., 2007).

Embora a hospitalização proporcione benefícios para o Estado de saúde da criança, esse processo pode provocar estresses e traumas (medos e inseguranças), os quais poderão ter como sequelas, por exemplo, o comprometimento do processo de desenvolvimento infantil (DOMINGUES; MARTINEZ, 2001). Bortolote e Brêtas (2008) acrescentam que quando a prática do profissional de saúde é realizada de forma impessoal, ou seja, sem considerar a criança com as suas particularidades e desenvolvimento, pode provocar atrasos nas aquisiçóes motoras e cognitivas.

As atividades cotidianas como comer, vestir, dormir, estudar, brincar, entre outras, caracterizam o lugar e o papel da criança na sociedade. $\mathrm{Na}$ hospitalização, a perda desse lugar e de papéis também é vivida como uma ruptura para a criança e sua família (TAKATORI; OSHIRO; OTASHIMA, 2004).

\subsection{O terapeuta ocupacional no hospital e as AVD}

É reconhecido na literatura que o terapeuta ocupacional, como membro de uma equipe interdisciplinar, contribui para a assistência da 
criança no hospital (TAKATORI; OSHIRO; OTASHIMA, 2004). Tal profissional busca destacar as capacidades da criança, contemplando-a como ser único e integro (MOTTA; TAKATORI, 2001). Estudando a importância da atuação da terapia ocupacional com a população infantil hospitalizada, Giardinetto et al. (2009) verificaram que segundo a visão dos profissionais da área da saúde, as crianças após a participaçáo nas atividades de terapia ocupacional, ficaram menos estressadas, assim como compreenderam e responderam melhor ao tratamento.

Dentre as funções do terapeuta ocupacional estão à promoçáo da autonomia e da qualidade de vida dos indivíduos, sendo que as AVD possuem um papel importante no investimento desse profissional junto às atividades presentes do cotidiano dos indivíduos (MATSUKURA; MARTURANO, 2001). De acordo com o Conselho Regional de Fisioterapia e Terapia Ocupacional da terceira região (2010), também compete ao terapeuta ocupacional planejar, prescrever e confeccionar adaptaçóes e dispositivos que auxiliam a realização de atividades, proporcionando independência, funcionalidade e qualidade de vida. A orientação e o treino relacionados a tais adaptaçóes e dispositivos também são atributos desse profissional.

Segundo Luzo, Mello e Capanema (2004, p. 111), "[...] o recurso tecnológico que possibilita a uma pessoa desempenhar uma atividade funcional é denominado assistivo." Nessa direção, a tecnologia assistiva caracteriza-se como conjunto de técnicas, aparelhos, instrumentos, produtos e procedimentos que objetivam facilitar a percepção, mobilidade e utilização do meio ambiente e dos elementos por pessoas com deficiência (ASSOCIAÇÃO..., 2004), idosos e pessoas com doença crônica (MELLO, 2008).

Discutindo sobre o uso da tecnologia assistiva no ambiente hospitalar Luzo, Mello e Capanema (2004) ressaltam as diferentes posturas exigidas dos profissionais quando prescrevem e selecionam equipamentos de autoajuda, pois no hospital há quadros agudos e crônicos, com ou sem instalação de incapacidades funcionais, dentre outras características, que exigem abordagens individualizadas. De acordo com os autores, o emprego da tecnologia asssitiva abrange todas as modalidades de desempenho humano, desde as atividades básicas de autocuidado até as atividades profissionais.

Para De Carlo, Bartalotti e Palm (2004), a terapia ocupacional vem direcionando suas açôes tanto nas conseqüências e no dia a dia da enfermidade e da internaçáo hospitalar, quanto nas diversas alteraçôes e rupturas que acometem os indivíduos. Contudo, observa-se na literatura as sugestôes de desenvolvimento e divulgaçáo de mais trabalhos na área, ressaltando a importância da compreensão das propostas e da inserção do terapeuta ocupacional neste campo de atuação (DOMINGUES; MARTINEZ, 2001; GIARDINETTO et al., 2009; PEDROSA et al., 2007).

Nesse sentido, cabe investigar de que forma a utilização de tecnologias se faz presente ou não no contexto hospitalar, a fim de possibilitar a discussão sobre o incremento de açóes relacionadas a esse tema, no contexto hospitalar. Diante deste contexto, justifica-se a necessidade e possibilidade de que novos conhecimentos sejam produzidos para a área de terapia ocupacional na hospitalizaçáo infantil e para a população que dele necessita.

\section{Objetivos}

Teve-se por propósito caracterizar a rotina de uma enfermaria pediátrica no que se refere ao desempenho de seus usuários, nas AVD: higiene pessoal, banho e alimentação; e apontar os possíveis usos da tecnologia assistiva relacionado ao desempenho dessas AVD.

\section{Método}

Trata-se de um estudo transversal, exploratório e descritivo, acerca das AVD de higiene pessoal, banho e alimentação de crianças internadas.

\subsection{Local}

A pesquisa foi desenvolvida em uma Enfermaria de Especialidades I e na Unidade de Cuidados Especiais de um Hospital Pediátrico de nível de atenção terciário, localizado na capital do estado de São Paulo.

\subsection{Participantes}

Participaram da pesquisa 10 membros de uma equipe de enfermagem, responsáveis pelos cuidados das crianças; 12 crianças internadas na instituição hospitalar de seis a doze anos; e seus respectivos pais ou responsáveis.

Os participantes foram convidados a participar da pesquisa, e na medida em que aceitaram, estes assinaram um Termo de Consentimento Livre e Esclarecido. Os pesquisadores também consideraram como critérios a vontade da criança em participar ou não da pesquisa. Como critérios de exlcusão, não foram participantes as crianças que a equipe médica e/ou de enfermagem considerou em condições 
clínicas inadequadas para a participação, assim como crianças com dificuldades intelectuais/cognitivas que impedissem a fidedignidade das respostas e a questão da independência nas AVD.

\subsection{Instrumentos da coleta de dados}

A caracterização da enfermaria pediátrica deu-se por meio de entrevistas semi-estruturadas e roteiros de observação, elaborados pelos pesquisadores, pois, a partir da revisão da literatura não foram encontrados instrumentos validados direcionados à coleta de dados sobre AVD no hospital com crianças, tampouco que fizessem relação com o uso da tecnologia assistiva neste contexto.

Segundo Minayo (2006), a entrevista, sempre que possível, deve ser acompanhada e complementada pela observação porque os dados provenientes do contexto da produçáo das falas proporcionam ao investigador adquirir elementos de relaçóes, práticas, cumplicidades, omissóes e imponderáveis que permeiam o cotidiano estudado.

Os instrumentos, de forma sucinta, abrangeram questôes relacionadas ao histórico da internação (por exemplo, se primeira internação, tempo de internação, como realiza as AVD; se a criança faz sozinha ou náo, quem a auxilia, quanto tempo demora, se há dificuldades, se há presença de equipamentos de autoajuda).

Especificamente para os pais, haviam itens que possibilitavam a comparação de como a criança realizava a atividade em casa e como conseguia desempenhar no hospital e a justificativa para tal mudança.

Já nos instrumentos direcionados aos membros da equipe de enfermagem, também se investigou como era a participação deles na realização das atividades com a criança (orientação e/ou supervisão e/ou execução).

\subsection{Procedimentos para análise dos dados}

Desse modo, a partir do conjunto de informaçôes obtidas da leitura do campo, da tabulação e cálculos com estatística descritiva simples, apresentada a partir de gráficos, discutiu-se a possibilidade da implantação e uso de dispositivos de tecnologia assistiva referente às AVD investigadas no contexto hospitalar, de modo à favorecer a independência destas com as crianças internadas, bem como auxiliar os profissionais de saúde na realização desse tipo de procedimento, conservando e diminuindo os gastos de energia dos envolvidos no seu desempenho.

\subsection{Aspectos éticos}

A pesquisa foi submetida e aprovada pelo Comitê de Ética em Pesquisa em Seres Humanos da Universidade Federal de São Carlos, sob o parecer $n^{\circ}$. 281/2011. Além desses procedimentos, a pesquisa também foi submetida à avaliação e autorização do responsável técnico da Enfermaria de Especialidades I e da Unidade de Cuidados Especiais do local da pesquisa. Esta avaliaçáo foi necessária para a realização da coleta de dados nesta instituição hospitalar de atendimento à criança.

\section{Resultados}

No total, foram entrevistados 10 (dez) profissionais da equipe de enfermagem, responsáveis pelos cuidados das crianças, 12 (doze) crianças de 6 (seis) a 12 (doze) anos internadas na Enfermaria de Especialidades I ou na Unidade de Cuidados Especiais e 12 (doze) responsáveis pelas crianças participantes. Além das entrevistas, realizaram-se observaçóes das AVD de higiene pessoal e/ou banho e/ou alimentação das crianças participantes.

\subsection{Sobre a utilização da tecnologia no hospital}

Como resultados, tem-se que 100\% ( $n=10)$ dos profissionais, membros da equipe de enfermagem referiram realizar orientaçáo e/ou supervisão e/ou execução das AVD de higiene pessoal, banho e alimentação das crianças sob sua responsabilidade; $100 \%(\mathrm{n}=10)$ relatam usar equipamentos de autoajuda para tais tarefas de acordo com os equipamentos e/ou dispositivos disponibilizados pela instituição hospitalar e a necessidade da criança; $100 \%(\mathrm{n}=10)$ afirmam que outros equipamentos ou dispositivos não disponíveis na instituição hospitalar podem contribuir para o seu trabalho; e $40 \%(n=4)$ reportaram não conhecer tais dispositivos ou equipamentos com a terminologia de "tecnologia assistiva".

\subsection{A independência nas AVD em contexto hospitalar- os relatos das crianças}

Entre as crianças participantes, o tempo de internação variou de três a quarenta e cinco dias; $75 \%(\mathrm{n}=9)$ referem dependência na AVD de higiene pessoal, $83,3 \%(\mathrm{n}=10)$ na atividade de banho 
e $16,6 \%(\mathrm{n}=2)$ na atividade de alimentação; as crianças relatam também que seus acompanhantes são quem as ajuda nas AVD de higiene pessoal e/ou banho e/ou alimentação.

Em relação ao uso de equipamentos e/ou dispositivos de auxílio para a execução das atividades perguntadas, as crianças que afirmaram utilizá-los $(50 \%, \mathrm{n}=6)$ citam as barras de apoio no banheiro e/ou cadeira de banho e/ou bandeja como exemplos.

A Figura 1 ilustra a porcentagem de crianças que referiram alguma dependência na realização das AVD de higiene pessoal e/ou banho e/ou alimentação:

\subsection{A independência nas AVD em contexto hospitalar- os relatos dos pais}

Os responsáveis pelas crianças participantes reportam que houve diferença no modo de execução da atividade de higiene pessoal $(41,6 \%, \mathrm{n}=5)$, de banho $(66,6 \%, n=8)$ e de alimentaçáo $(50 \%, n=6)$ das mesmas. Embora com pequenas diferenças dos relatos das crianças, os pais reportaram dados similares, sendo o banho a atividade com maior porcentagem de ajuda, conforme a Figura 2.

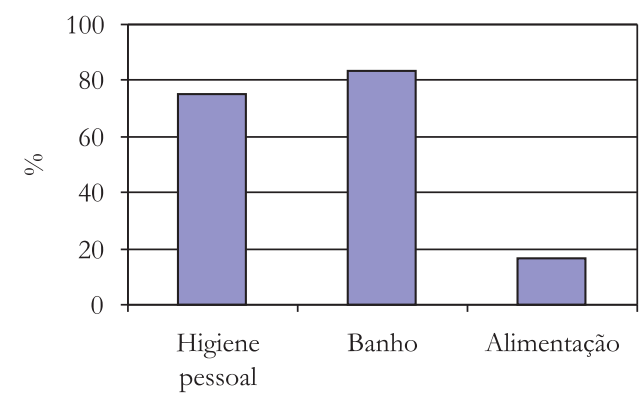

Figura1. Crianças que referiram alguma dependência na realização das AVD de higiene pessoal e/ou banho e/ou alimentação.

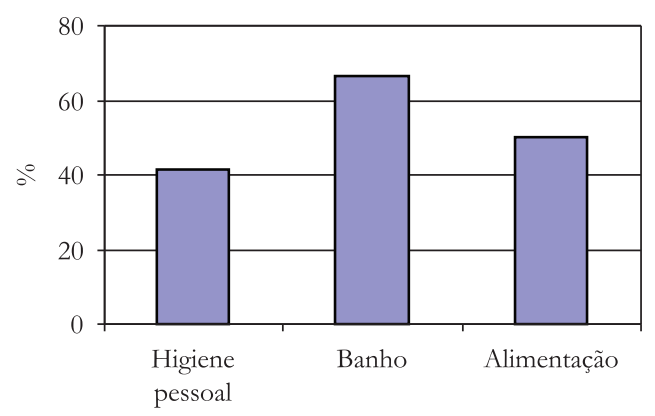

Figura 2. Atividades das crianças que os responsáveis relataram diferença no modo de execução em casa e no hospital.
Cabe destacar que das crianças com mudança na realização da AVD de higiene pessoal, $60 \%(\mathrm{n}=3)$ dos responsáveis identificam como causa a doença, $20 \%(\mathrm{n}=1)$ a internação hospitalar e $20 \%(\mathrm{n}=1)$ ambas as condiçôes (doença e internação hospitalar); no banho, $62,5 \%(\mathrm{n}=5)$ dos responsáveis identificam como causa a doença, $25 \%(\mathrm{n}=2)$ a internação hospitalar e $12,5 \%(n=1)$ ambas as condiçóes (doença e internação hospitalar); e na alimentação, $50 \%(\mathrm{n}=3)$ dos responsáveis identificam como causa a doença, 33,3\% ( $\mathrm{n}=2)$ a internação hospitalar e $16,7 \%(n=1)$ ambas as condiçôes (doença e internação hospitalar).

A Figura 3 ilustra o motivo da condição de mudança na execução das AVD de higiene pessoal e/ou banho e/ou alimentação da crianças segundo seus responsáveis.

No total, 41,6\% $(\mathrm{n}=5)$ dos responsáveis afirmam que a criança sob sua responsabilidade faz uso de equipamentos e/ou dispositivos de auxílio nas atividades de higiene pessoal e/ou banho e/ou alimentação.

\section{Discussão}

Os dados coletados corroboram com a literatura ao demonstrar que as crianças, durante o período de internação, vivenciam a mudança do espaço físico (do lar para o hospital), procedimentos que podem causar medo e emoçóes de sofrimento (desde condutas terapêuticas até a atividade de banho quando há dores intrínsecas da sintomatologia do quadro clínico), ruptura do cotidiano, entre outros (DOMINGUES; MARTINEZ, 2001; TAKATORI; OSHIRO; OTASHIMA, 2004).

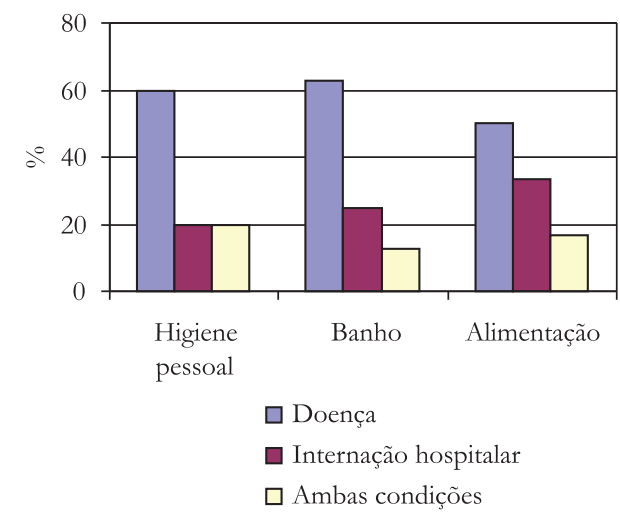

Figura 3. Motivo da diferença no modo de execução das atividades pelas crianças na opinião dos responsáveis. 
Desta maneira, tem-se que a mudança na condição de sujeitos independentes para sujeitos dependentes e/ou a alteração no modo de fazer as AVD de higiene pessoal e/ou banho e/ou alimentação, mesmo que temporariamente, é vivenciada por estas crianças. Fatores como a fase da patologia e tratamento, a estrutura física (divisão de leitos por quarto, banheiro e outros ambientes coletivos e individuais) e a dinâmica hospitalar (horários das refeiçóes e da visita médica, p. ex.) influenciam no desempenho das AVD das crianças no hospital. Além disto, tais fatores exemplificam a alteração na rotina familiar até então conhecida pela criança, pois se tem que a maioria das AVD são programadas a partir dos cuidados dirigidos à patologia e da rotina preestabelecida pela instituição hospitalar (MORSCH; ARAGÃO, 2008).

Destaca-se ainda que os responsáveis das crianças internadas, com a intenção, por exemplo, de minimizar os impactos da hospitalização, auxiliam e/ou fazem pela criança as AVD de higiene pessoal (p. ex.: escovar os dentes), banho (p. ex.: ensaboam o corpo da criança) e alimentação (p. ex.: dar a comida na boca) das crianças, sendo que as mesmas conseguiriam realizá-las de maneira independente no contexto hospitalar mesmo que demandando um tempo maior.

Destaca-se aqui outro fator relevante nos cuidados prestados às crianças durante o período de hospitalização, trata-se da participação de pessoas de referência primária (como os pais, avôs e tios, p. ex.) no auxilio de tais atividades, sabendo que experiências de segurança interna e confiança influenciam na estrutura psíquica e na qualidade de vida da criança internada (MORSCH; ARAGÃO, 2008).

Durante a coleta de dados, observou-se que os dispositivos de tecnologia assistiva direcionados para as AVD de higiene pessoal, banho e alimentaçáo disponíveis são limitados e encontraram-se apenas os mais básicos do contexto hospitalar, tais como a cadeira de banho, as barras de apoio e/ou para transferências e uma bandeja para alimentação. Supóe-se que por meio da inserçáo de novos equipamentos e dispositivos de tecnologia assistiva, como banco, para o banho, um suporte de cabo alongado para bucha, copos recortados e com alças e talheres engrossados ou flexíveis, as crianças, em hipótese poderiam ter maior independência durante o período de internação e, tanto os profissionais quanto os responsáveis, teriam maior segurança em permitir o desempenho das AVD pelas crianças de modo independente, sem sua participação.

\subsection{Possíveis usos da tecnologia na enfermaria pediátrica, relacionados às AVD: implicações}

Considerando a complexidade e o número de fatores envolvidos na prescrição de equipamentos de tecnologia assistiva aos usuários no contexto hospitalar, observa-se a necessidade do envolvimento multidisciplinar (LUZO; MELLO; CAPANEMA, 2004), a fim de que alguns itens como segurança (para prevenir lesões ou perda de função), design simples, tamanho ajustável, conforto, fácil aplicação e remoção, manutenção e higiene, sejam apreciados (CAVALCANTI; GALVÃO, 2007).

$\mathrm{Na}$ situação de hospitalização, têm-se casos de perda funcional transitória, como é o caso, por exemplo, de algumas das crianças participantes deste estudo. Nestas situaçóes, a abordagem deve direcionar-se no aumento da independência e da autonomia do paciente nas atividades de autocuidado prejudicadas (LUZO; MELLO; CAPANEMA, 2004).

Deste modo, o terapeuta ocupacional deve atuar de forma mais integrada possível com a equipe de enfermagem, pois, como se observou nos dados coletados, os principais responsáveis pela orientação ou supervisão ou desempenho das AVD das crianças internadas são os membros da equipe de enfermagem.

Assim, os mesmos devem ser informados quanto ao uso de equipamentos de tecnologia assistiva prescritos aos pacientes e/ou disponíveis na instituição e quanto à importância do incentivo a sua independência e autonomia (LUZO; MELLO; CAPANEMA, 2004).

Para Luzo, Mello e Capanema (2004), idealmente, o setor de terapia ocupacional deveria disponibilizar, por meio de empréstimos e/ou locação, os equipamentos de tecnologia assistiva aos pacientes, visando atender imediatamente às necessidades dos mesmos, e orientar a aquisição de tais equipamentos apenas quando o uso for prolongado.

Para as AVD de higiene pessoal e de banho, os equipamentos de tecnologia assistiva, ampliam o desempenho e a funcionalidade da criança, visando proporcionar condiçóes de segurança e conforto e, muitas vezes, a seleção de determinada adaptação é respaldada nos critérios de acessibilidade, proteçáo articular e conservação de energia (CAVALCANTI; GALVÃO, 2007).

Citam-se como possíveis intervençóes efetivas no banheiro instalaçóes de barras de segurança e transferência, introduçáo de banco ou cadeira para banho, bucha com cabo curvo alongado e engrossador universal para acoplar objetos como escova de dente, escova de cabelo, dentre outros. 
Refletindo sobre a adaptação da estrutura física, observou-se que, especificamente no local da pesquisa, a presença de banheiros pequenos e que dificultavam tanto a usabilidade quanto a mobilidade dos indivíduos no mesmo. Por isso, pensando na modificação do ambiente, sugerem-se modificaçôes embasadas nos parâmetros construtivos da norma NBR-9050, que indicam diretrizes para o planejamento de edificaçóes, mobiliário e equipamentos com equidade, independência, conforto e segurança.

$\mathrm{Na}$ AVD de alimentação, os equipamentos de tecnologia assistiva proporcionam a ampliação da capacidade de manter e manipular utensílios e conduzir o alimento (sólido ou líquido) à boca (CAVALCANTI; GALVÃO, 2007).

Citam-se como possíveis equipamentos para tal atividade a utilização de bandeja com suporte para copo e, se necessário, utilizaçáo de um prato com ventosa para a fixação do mesmo na bandeja, pratos com borda elevada, talheres com cabo alongado, engrossado ou com proteção plástica, copos com tampa em bico, com tampa e canudo, de alça uni ou bilateral, copo com recorte ou de peso no fundo.

Cabe mencionar que as características particulares de cada instituição hospitalar e da clientela atendida devem ser compreendidas e analisadas antes da compra ou inserçáo de tais equipamentos na rotina dos profissionais e dos pacientes, pois poderá acontecer destes recursos não serem uma demanda para determinada instituição devido as suas especificidades.

\section{Considerações finais}

A partir dos dados coletados e dos referenciais teóricos que embasam este trabalho, foi possível documentar informaçóes referentes ao desempenho nas AVD de higiene pessoal, banho e alimentação de crianças de 6 a 12 anos internadas, se havia ou náo o uso de algum dispositivo de autoajuda para desempenho destas e como estes se encontravam inseridos no contexto hospitalar.

Este estudo possibilitou registrar a percepção dos principais sujeitos envolvidos- crianças, responsáveis e equipe de enfermagem- sobre a maneira que estes desenvolvem tais atividades no hospital.

Verificou-se a potencialidade da tecnologia assistiva na promoção da independência nas AVD de crianças internadas e a possibilidade de sugestóes e discussôes de recursos mais adequados às condiçóes estudadas.

Este estudo, ainda que em uma dimensão reduzida, se considerar que ocorreu em um único local e que não houve a implementação dos dispositivos de tecnologia assistiva no contexto, colaborou para o conhecimento produzido na área e seus resultados e discussão podem contribuir para que profissionais possam refletir sobre suas práticas junto a crianças internadas.

Observa-se que a temática estudada é de relevância acadêmica e prática, pois na literatura científica ainda são escassos os artigos publicados e indexados online. Do mesmo modo, é importante salientar a necessidade de mais estudos na área e da divulgação e publicação de trabalhos realizados por terapeutas ocupacionais neste contexto a fim de oferecer benefícios à população atendida e contribuir para o crescimento e fortalecimento da profissão.

\section{Referências}

ASSOCIAÇÃO BRASILEIRA DE NORMAS TÉCNICAS - ABNT. NBR 9050: acessibilidade a edificaçôes, mobiliário, espaços e equipamentos urbanos. Rio de Janeiro: ABNT, 2004.

BEE, H. O ciclo vital. Tradução de Regina Garcez. Porto Alegre: Artmed, 1997. $656 \mathrm{p}$.

BORTOLOTE, G. S.; BRÊTAS, J. R. S. O ambiente estimulador ao desenvolvimento da criança hospitalizada. Revista da Escola de Enfermagem da USP, São Paulo, v. 42, n. 3, p. 422-429, set. 2008. Disponível em: <http:// www.scielo.br/scielo.php?script=sci_arttext\&pid=S0080$62342008000300002 \& I n g=p t \& n r m=i s o>$. Acesso em: 01 dez. 2009. http://dx.doi.org/10.1590/ S0080-62342008000300002

BRASIL. Ministério da Saúde. Secretaria de Políticas de Saúde. Departamento de Atençâo Básica. Saúde da criança: acompanhamento do crescimento e desenvolvimento infantil. Brasília: Ministério da Saúde, 2002.

BRONFENBRENNER, U. A Ecologia do Desenvolvimento Humano: experimentos naturais e planejados. Porto Alegre: Artes Médicas, 1996.

CAVALCANTI, A.; GALVÃO, C. Adaptação ambiental e doméstica. In: CAVALCANTI, A.; GALVÃO, C. Terapia Ocupacional: fundamentação e prática. Rio de Janeiro: Guanabara Koogan, 2007. p. 420-426.

CONSELHO REGIONAL DE FISIOTERAPIA E TERAPIA OCUPACIONAL DA TERCEIRA REGIÂO - CReFiTo-3. Terapia Ocupacional: definiçāo. Disponível em: <http://www.crefito.com.br/> . Acesso em: 15 abr. 2010.

DE CARLO, M. M. R. P.; BARTALOTTI, C. C.; PALM, R. D. C. M. A terapia ocupacional em reabilitação física e contextos hospitalares: fundamentos para a prática. In: DE CARLO, M. M. R. P.; LUZO, M. C. M. Terapia ocupacional: reabilitação física e contextos hospitalares. São Paulo: Roca, 2004. p. 3-28.

DOMINGUES, A. C. G.; MARTINEZ, C. M. S. Hospitalização infantil: buscando identificar e caracterizar experiências de terapia ocupacional com crianças 
internadas. Cadernos de Terapia Ocupacional da UFSCar, São Carlos, v. 9, n. 1, p. 16-29, 2001.

GIARDINETTO, A. R. S. B. et al. A importância da terapia ocupacional com a população infantil hospitalizada: a visão de profissionais da área da saúde. Cadernos de Terapia Ocupacional da UFSCar, São Carlos, v. 17, n. 1, p. 63-69, jan./jun. 2009.

GRIGOLATTO, T. et al. A intervenção terapêutica ocupacional em CTI pediátrico: um estudo de caso. Cadernos de Terapia Ocupacional da UFSCar, São Carlos, v. 16, n. 1, p. 37-46, jan./jun. 2008.

LINHARES, M. B. M. et al. A compreensão do fator de risco da prematuridade sob a ótica desenvolvimental. In: MARTURANO, E. M.; LINHARES, M. B. M.; LOUREIRO, S. R. Vulnerabilidade e proteção indicadores na trajetória de desenvolvimento do escolar. São Paulo: Casa do Psicólogo; FAPESP, 2004. p. 10-38.

LUZO, M. C. M.; MELLO, M. A. F.; CAPANEMA, V. M. Recursos tecnológicos em terapia ocupacional - órteses e tecnologia assistiva. In: DE CARLO, M. M. R. P.; LUZO, M. C. M. Terapia ocupacional: reabilitação física e contextos hospitalares. São Paulo: Roca, 2004. p. 99-126.

MATSUKURA, T. S.; MARTURANO, E. M. Catálogo de avaliação de independência de crianças de 4 a 8 anos nas atividades de vida diária. São Carlos: Edufscar, 2001. 25 p. (Série Apontamentos).

MELLO, M. A. F. A tecnologia assistiva no Brasil. In: OLIVEIRA, A. I. A.; LOURENÇO, J. M. Q.; LOURENÇO, M. G. F. Perspectivas da tecnologia assistiva no Brasil: pesquisas e práticas. Belém: EDUERA, 2008. p. $7-14$.
MELLO, M. A. F.; MANCINI, M. C. Métodos e técnicas de avaliação nas áreas de desempenho ocupacional. Seção 9.1 - Avaliaçóes das atividades de vida diária e controle domiciliar. In: CAVALVCANTI, A.; GALVÃO, C. Terapia ocupacional: fundamentação e prática. Rio de Janeiro: Guanabara Koogan, 2007. p. 49-54.

MINAYO, M. C. S. O desafio do conhecimento: pesquisa qualitativa em saúde. 9. ed. São Paulo: Hucitec, 2006.

MORSCH, D. S.; ARAGÃO, P. M. A criança, sua família e o hospital: pensando processos de humanização. In: DESLANDES, S. F. Humanização dos Cuidados em Saúde: conceitos, dilemas e práticas. Rio de Janeiro: Editora FIOCRUZ, 2008. p. 235-260.

MOTTA, M. P.; TAKATORI, M. A assistência em terapia ocupacional sob a perspectiva do desenvolvimento da criança. In: DE CARLO, M. M. R. P.; BARTALOTTI, C. C. Terapia Ocupacional no Brasil: fundamentos e perspectivas. São Paulo: Plexus, 2001. p. 117-135.

PEDROSA, A. M. et al. Diversão em movimento: um projeto lúdico para crianças hospitalizadas no Serviço de Oncologia Pediátrica do Instituto Materno Infantil Prof. Fernando Figueira. Revista Brasileira de Saude Materno Infantil, Recife, v. 7, n. 1, p. 99-106, jan./mar. 2007. http://dx.doi.org/10.1590/S1519-38292007000100012 TAKATORI, M.; OSHIRO, M.; OTASHIMA, C. O hospital e a assistência em terapia ocupacional com a populaçáo infantil. In: DE CARLO, M. M. R. P.; LUZO, M. C. M. Terapia ocupacional: reabilitação física e contextos hospitalares. São Paulo: Roca, 2004. p. 256-275.

TEIXEIRA, E. Atividades da vida diária. In: TEIXEIRA, E. et al. Terapia Ocupacional na Reabilitação Física. São Paulo: Roca, 2003. p. 193-219.

\section{Contribuição dos Autores}

Adriana Moniz da Silveira: Concepção do texto manuscrito; organização de fontes e/ou análises, redação do texto, revisão. Regina Helena Vitale Torkomian Joaquim: Concepção do texto manuscrito; organização de fontes e/ou análises, redação do texto, revisão. Daniel Marinho Cezar da Cruz: Auxiliou na análise, discussão e revisão dos resultados. 